\title{
CORRECTION
}

\section{Correction: Rational fluid management in today's ICU practice}

\author{
Karsten Bartels', Robert H Thiele ${ }^{2}$ and Tong J Gan*1 \\ See related review by Bartels et al., http://ccforum.com/content/17/S1/S6
}

\section{Correction}

Our recently published review article [1] contained a misprint. On page four under the sub-heading 'Goaldirected fluid therapy' and in the third paragraph, the sentence:

"Results of a randomized trial investigating mortality in 3,141 children with severe febrile illness and impaired perfusion in sub-Saharan Africa surprisingly showed higher mortality at 48 hours and at 4 weeks in the group that did not receive any fluid boluses compared with two groups resuscitated with albumin or saline [38]."

should have instead read:

"Results of a randomized trial investigating mortality in 3,141 children with severe febrile illness and impaired perfusion in sub-Saharan Africa surprisingly showed lower mortality at 48 hours and at 4 weeks in the group that did not receive any fluid boluses compared with two groups resuscitated with albumin or saline [38]."
The authors sincerely regret this error.

\section{Competing interests}

TJG has received research funding from Fresenius Kabi and honoraria from Hospira and Baxter. KB and RHT declare that they have no competing interests.

\section{Author details}

'Department of Anesthesiology, Box 3094, Suite 5670B, Duke University Medical Center, Durham, NC 27710, USA. ${ }^{2}$ Department of Anesthesiology, University of Virginia School of Medicine, PO Box 800710, Charlottesville, VA 22908-0710, USA.

Published: 26 June 2013

\section{Reference}

1. Bartels K, Thiele R, Gan T: Rational fluid management in today's ICU practice. Critical Care 2013, 17(Suppl 1):S6.

doi:10.1186/cc12771

Cite this article as: Bartels K, et al: Correction: Rational fluid management in today's ICU practice. Critical Care 2013, 17(Suppl 1):S10.
*Correspondence: tong.gan@dm.duke.edu

'Department of Anesthesiology, Box 3094, Suite 5670B, Duke University Medical Center, Durham, NC 27710, USA

Full list of author information is available at the end of the article 